\title{
Learning Robots: Teaching Design Students in Integrating Intelligence
}

\author{
Emilia Barakova and Jun $\mathrm{Hu}$ \\ Department of Industrial Design, \\ Eindhoven University of Technology \\ \{e.i.barakova, j.hu\}@tue.nl
}

\begin{abstract}
The present day society requires specialists with multidisciplinary knowledge and skills. We discuss the possibilities to educate professionals that design intelligent products and systems as a result of a competency based education. In particular this paper features a teaching method that makes the students use intelligent algorithms that control robot behavior in a way that the robot can solve design problems with practical relevance, and reach human to machine interaction that includes machine intelligence. The outcomes of 14 cases done over 4 years showed that most students were able to integrate the required competencies in a synergistic way. Within 80 hours they could master the technical complexity of controlling a robot through a neural learning algorithm in well-grounded design cases.
\end{abstract}

Keywords: competency based learning, learning algorithms, behavioral robotics, adaptive design applications.

\section{Introduction}

Societal, scientific and technological developments are changing the field of industrial design. The field expands towards designing for intelligent systems, products and related services. If one truly likes to design systems that express intelligence in societally meaningful way, we believe that it implies a novel view on education that prepares specialists that can use skills and knowledge from different disciplines in a synergistic manner. The Department of Industrial Design, Eindhoven University of Technology, has been developing an educational program that will meet this challenge. In order to do so, the department offers a self-directed, competency-centered education model, including both: competency framework and the reflective transformative design process, in which learning and creating come together. We facilitate the learning process to let the students develop the ability to deal creatively and flexibly with the large amounts of constantly evolving information in globalized knowledge economy [1-4].

Designing embodied intelligent products is a contemporary challenge which deserves more attention and better support by novel methods and tools. For a long time the worlds of artificial embodied intelligence (represented by behavioral robotics) and industrial design developed separately. The intelligent objects were not developed to 
be used by real users, they were merely a research playground. In addition, behavioral robotics was mostly occupied with constitutive aspects of autonomy, such as collision avoidance, search for energy sources, and survival, and barely with interaction with humans. Examples of products that have embodied intelligence are robotic vacuum cleaners and grass trimming machines. In contrast, the industrial designers had to give high priority to interaction and usability, to emotional associations, styling, appeal, and even fun associated with the product. At present, the need for embodied intelligence features becomes apparent in many everyday personal objects and as a consequence, the two disciplines are merging. At the methodological level however, much work regarding their integration remains to be done [5].

The competency based learning framework gives equal weight to knowledge, skills and attitudes. Differently from the traditional studies, the knowledge, skills and attitudes are integrated already during the university education. This integration requires a powerful and rich learning environment. The student has to learn how to model contexts of use, how to actively explore concepts, how to evaluate alternative solutions, how to bring new artifacts into the world, in other words, how to design. This learning model appears not only to be well-accepted for traditional industrial design, where the material form of things is the central theme, but also to be effective for the aspects of industrial design that is concerned with creation of dynamic form (i.e. when the behavior of the product is designed on many levels).

Facing these challenges, this paper features a teaching method that makes the students combine artificial intelligence methods, in particular neural learning algorithms, to solve design problems. First, in the competency based educational system that aims at specialists with balanced knowledge of the modern technologies, user-centered design, design of business processes and conventional design education are combined. Second, we chose the very core of this education - designing of intelligent products and explore the importance of concepts such as embodiment, emergence, interactivity and learning within meaningful design applications. We analyze the outcomes of fourteen projects and show what the drawbacks are in the teaching process, in attempt to find new recommendations of how to prepare students for complex and multidisciplinary realities of the future.

\section{Technology and Intelligence in Competency Based Learning}

\subsection{Competence Framework}

At our department, the competency based learning framework includes ten specific competency areas that are involved during designing, related to the content of the system, product or service to be designed, and/or to the approach needed for the act of designing or becoming a designer [3, 4]. These ten competency areas are: 1) self-directed and continuous learning, 2) descriptive and mathematical modeling, 3) Integrating technology, 4) ideas and concepts, 5) form and senses, 6) user focus and perspective, 7) social and cultural awareness, 8) designing business processes, 9) design and research processes, 10) teamwork and communication. In this framework, 
- Self-Directed and Continuous Learning is about taking responsibility for and give direction to your own personal development, based on a continuous process of selfreflection and out of curiosity for future developments in technology and society;

- Descriptive and Mathematical Modeling is about being able to create and apply models by using formal and mathematical tools, in order to justify design decisions and support the design of complex, highly dynamic and intelligent systems;

- Integrating Technology is about being competent in exploring, visualizing, creating and demonstrating innovative concepts and experiences using technology, as well as analyzing the technical and economic feasibility of complex designs in which technology is integrated. Moreover, one needs to understand scientific papers and be able to communicate with engineers and researchers of another disciplines;

- Ideas and Concepts is about developing visions, innovative ideas and concepts through creativity techniques, experimentations and the translation of research.

- Form and Senses is about Experiencing and developing through doing and abstraction, aesthetical (physical) languages that connect thought and interactive form, in order to communicate specific properties of the design concept.

The aim of the study is to make the students develop these competencies in a synergistic way: the students have to not only gain proficiency in the separate competency, but to integrate them into a novel quality. Integration is done through practically oriented activities such as projects and assignments in which equal weight is given to knowledge, skills and attitudes, and stimulates students to learn by doing [3, 4]. It is about learning and performing through practical application, while simultaneously acquiring theoretical skills, to the level that the students are participating in the research of the department. Both aspects are bridged through reflection on the taken actions. This approach fits the profession of industrial designer perfectly. Consequently, the design process we offer the students should be holistic and give equal weight to knowledge, skills and attitudes throughout the process and stimulate reflection.

\subsection{Course Design}

Designing intelligent products involves creation and interpretation of meanings through form and behavior. Apart from functional aspects of meanings there are very important non-functional aspects such as creating affordances, contexts and experiences [6]. There are static as well as dynamic aspects that facilitate affordances, contexts and experiences creation. While static refers to the form, shape, material, color and texture of the artifact, dynamic refers to behavior, in other words, the movement, light and sound, and the changes of form, shape, material, color and texture that take place over time. All the mentioned aspects are, however, non-separable, which can very well be explained with the notion of embodiment. In the past few decades there was a converging interest in embodiment from scholars in philosophy, cognitive science, psychology, linguistics, robotics, and neuroscience. Embodiment signifies that the static or physical aspects of the product determine and provoke bodily interactions with the surrounding world. For simple products these interactions are rather passive and simple to anticipate. However, nowadays products are starting to behave, have autonomy, intelligence, and even emotions [7-13]. 
To cope with these novel requirements of the product design we have introduced a multidisciplinary course called Learning robots that focuses on usage of braininspired learning algorithms, implemented on an embodied agent (robot or a tangible interaction tool) within an interaction design task. In terms of the competence based educational system, the following competencies were addressed: integrating technology, that accounts for the technical implementation of the learning algorithms on embodied agents; mathematical modeling that embeds the learning process within the selected design task; form and senses, for designing the particular embodiment, the behavior, and the interaction patterns of the robots.

The tools and platforms used within this course were a selected set of learning algorithms that were implemented on Matlab and explained to the students on the level of concept, working and implementation. Two robotic platforms, namely AdMoVeo robot [14] and i-blocks [15] were used as a platforms to develop interaction behaviors and Neuroph neural learning engine was provided [18].

\subsection{Embodiment: AdMoVeo Robot}

To teach the students learning algorithms and programing skills, the focus shall not be distracted by form giving and embodiment. Using robots in this context has been a positive experience in our teaching practice $[5,16]$. The AdMoVeo robot (Fig. 1) is designed especially for this purpose $[14,17]$.

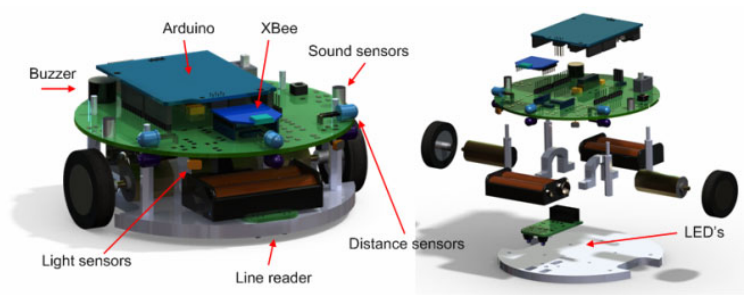

Fig. 1. 3D rendering of the AdMoVeo robot

The design of AdMoVeo features a detachable Arduino Diecimila board and two wheels integrated within the round shape of the chassis. The chassis and motor mount are made from transparent acryl glass, giving it a see-through look into everything inside. The sensors include two line readers at the bottom, three infrared distance sensors at the sides and in the front with sensibility of 0 to $20 \mathrm{~cm}$, two light sensors in the front, two sound sensors at the sides and two optional encoders coupled to wheels. The actuators include two motors driving two wheels, a buzzer and a RGB color LED integrated into the acryl chassis. An XBee module is optional for wireless communication. The software design is based on a layered structure of composition and inheritance. It has mainly two major parts: the firmware IDuino running in the Arduino microcontroller of the AdMoVeo robot, and the Java API library for programming and controlling AdMoVeo in the Processing programming environment [17]. Processing is an open source programming language and environment widely used by artists, designers and researchers to program images, animation, and interactions. 
To easily implementing some of the learning algorithms and interfacing with the AdMoVeo robot through Java-based Processing, students were encouraged to use Neuroph $^{1}$, a lightweight Java neural network framework [18]. Some groups used MATLAB.

\section{Design Cases}

During 4 years 14 groups of 3 to 4 students were working on different design cases. The students were thought 3 different learning paradigms and possible applications were discussed and demonstrated for each algorithm. The students were introduced to the robotic platforms and they also could choose to make an own platform. Each hardware platform had to perform an intelligent behavior caused by a learning algorithm. The behaviors had to be a solution to a design case in which learning will be of true added value. In the following subsections we introduce 2 outcomes of student's modules that serve as an illustration for the analysis of the overall outcomes of this learning activity with respect to competencies development and integration.

\subsection{Training Robot Dog}

In this design case a robot was trained as a pet-dog that is rewarded for accomplishing of a meaningful action. In this case the advanced interaction possibilities between a robot and a human were addressed. The complexity of this task comes from the definition of "meaningful" action. An action that is meaningful to human usually consists of more than one simple action. Simple action is known in behavioral robotics as action or movement primitive, and a typical reinforcement learning algorithm could easily learn to reward such primitives. In the case of a meaningful action, the reinforcement has to come after different number of primitive actions.

The experimental setting consisted of 2 steps. First, supervised learning was used for the recognition of spoken commands that give order to the robot dog. And second, the Q-learning algorithm was used to encourage the performed action sequences. Initially the robot responds to a voice command with a random action. The action was rewarded or punished each time by the user and the robot remembered the association between a reward and a meaningful action. Next time the robot hears the command it will try to perform the proper action to receive a reward.

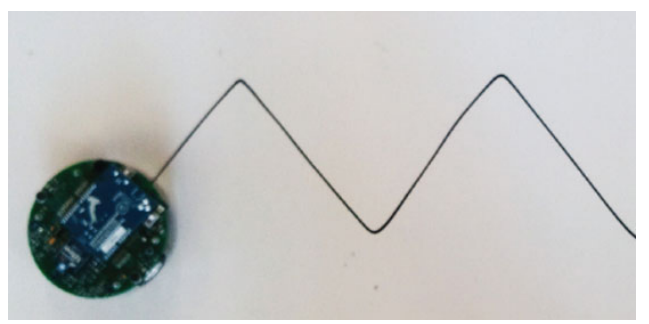

Fig. 2. Design case: training a robot dog

\footnotetext{
${ }^{1}$ neuroph.sourceforge.net
} 


\subsection{Fashion Business}

The Fashion business case features a robot assistant that consisted from the AdMoVeo robot, augmented with a web camera that is able to guide people through a clothing store and to suggest clothing that fits them (Fig. 3). The robot was able to dynamically adapt towards new clothing in a shop, analyze customers clothing and learn to give appropriate suggestions from feedback of the customers. The robot was designed to use a feedback from the color of the item that the previous customers bought and to link that with the clothing used by these customers. Information from different customers was continuously updated with the aim that the robot also can recognize for example the most popular clothing.
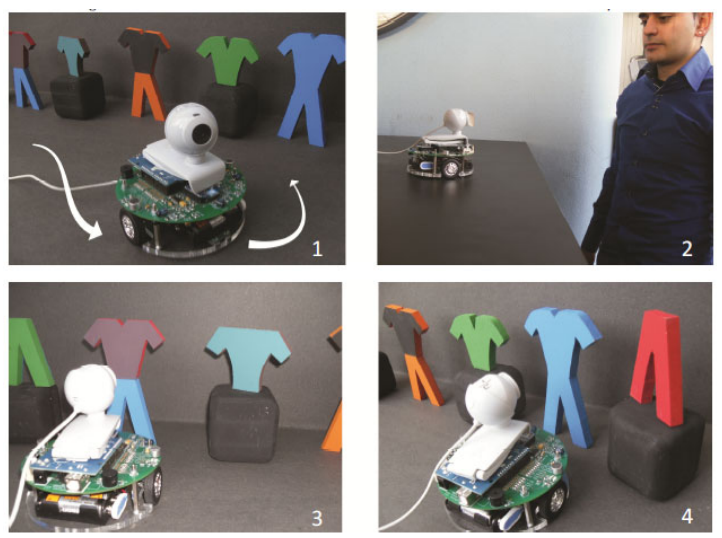

Fig. 3. Design case: Fashion business

Behavioral scenario consisted from two phases. First, the robot explored the shop to identify different available colors of clothing. The neural gas algorithm that uses a number of predefined classes was used for this application. With this algorithm several classes where created on the basis of RGB values of different captured colors. After training the classes were fixed.

In the second phase, (Fig. 3, capture 2), a new customer enters the shop. The average color of his/her clothes is captures and translated into RGB values, which serve as an input to the learning algorithm which classifies it to the clusters of colors that were fixed at phase one. The robot will then be able to make suggestions according to the colors that a customer is wearing.

Within the time span of 60 to 80 hours the students were able to either create a good design framework and interaction scenarios and partially working solution, as in Design case 2, or to make well working application with a hypothetical real life application, as in the Design case 1. Three groups made their own hardware platform and controlled it with a learning algorithm to express an intelligent behavior. One group used the existing i-blocks, and 9 groups used AdMoVeo robot, and one group presented advanced computer simulation and a game design concept which were not integrated. Overall the three competencies were integrated within a coherent outcome. A single competency was dominant in each group. This depended on the learning 
goals of the students within the group. Moreover, students with similar level of development of their competency profiles and proficiency tended to work together in a group.

\section{Discussion}

This paper presents our practical experience with applying the competency based learning to educational topics that required multidisciplinary knowledge and skill development. There were three relatively complex tasks that had to be integrated in this module: original design concept, proficiency with using robotic hardware platform and creation of intelligent behavior of this platform within the design context. The outcomes over 4 years and 14 projects showed that the integration of the competencies that were needed for each design case, namely idea generation, integrating technology, form and senses, and mathematical and descriptive modeling were integrated in a synergistic way in most of the design cases outcomes. The few cases that lacked either strong real-life design application or working learning behaviors were due to too ambitious task choices.

Comparing these achievements with students that made long-term master projects on behavioral robotics within traditionally set education system, we found out that the abilities of the students educated within the competency based system differ with their determination to accomplish a societally meaningful and working application. Our master students can combine and use knowledge from several different disciplines in a synergistic and practically meaningful way. Sensing the new realities, gradually more students join ID education than traditional robotics related studies.

\section{References}

1. Hummels, C., Frens, J.: Designing for the unknown: A design process for the future generation of highly interactive systems and products 4, 204-209 (2008)

2. Hummels, C., Frens, J.: The reflective transformative design process. In: CHI 2009, pp. 2655-2658. ACM, Boston (2009)

3. Hummels, C., Vinke, D.: Eindhoven Designs, vol. 2. Eindhoven University of Technology, Edinhoven (2009)

4. Hummels, C., Vinke, D., Frens, J., Hu, J.: Competency-centered Education for Designing Interactive and Intelligent Products. Creation and Desion (to appear, 2011)

5. van der Vlist, B., van de Westelaken, R., Bartneck, C., Hu, J., Ahn, R., Barakova, E., Delbressine, F., Feijs, L.: Teaching Machine Learning to Design Students. In: Pan, Z., Zhang, X., El Rhalibi, A., Woo, W., Li, Y. (eds.) Edutainment 2008. LNCS, vol. 5093, pp. 206217. Springer, Heidelberg (2008)

6. Feijs, L.M.G., Barakova, E.I.: Semantics through embodiment: a non-linear dynamics approach to affective design. In: Feijs, L., Kyffin, S., Yong, B. (eds.) Design and Semantics of Form and Movement, pp. 108-116 (2007)

7. Barakova, E.I.: Emergent behaviours based on episodic encoding and familiarity driven retrieval. In: Bussler, C.J., Fensel, D. (eds.) AIMSA 2004. LNCS (LNAI), vol. 3192, pp. 188-197. Springer, Heidelberg (2004) 
8. Barakova, E., Lourens, T.: Expressing and interpreting emotional movements in social games with robots. Personal and Ubiquitous Computing 14, 457-467 (2010)

9. Barakova, E.I., Vanderelst, D.: From spreading of behavior to dyadic interaction - A robot learns what to imitate. International Journal of Intelligent Systems 26, 228-245 (2011)

10. Lourens, T., Barakova, E.: My Sparring Partner Is a Humanoid Robot. In: Mira, J., Ferrández, J., Álvarez, J., de la Paz, F., Toledo, F. (eds.) IWINAC 2009. LNCS, vol. 5602, pp. 344-352. Springer, Heidelberg (2009)

11. ten Bhömer, M., van der Aalst, K., Barakova, E., Ross, P.: Product adaptivity through movement analysis: the case of the intelligent walk-in closet. In: Proceedings of Design and Semantics of Form and Movement (DeSForM 2009), pp. 114-121. Koninklijke Philips Design, Taipei (2009)

12. Barakova, E.I.: Social interaction in robotic agents emulating the mirror neuron function. In: Mira, J., Álvarez, J.R. (eds.) IWINAC 2007. LNCS, vol. 4528, pp. 389-398. Springer, Heidelberg (2007)

13. Abraham, A., Jónyer, I., Barakova, E.I., Jain, R., Jain, L.: Special issue on hybrid neurocomputing. Neurocomputing 61, 1-3 (2004)

14. Hu, J., Alers, S.: AdMoVeo: Created For Teaching Creative Programming. In: Hirashima, T., Mohd Ayub, A.F., Kwok, L.-F., Wong, S.L., Kong, S.C., Yu, F.-Y. (eds.) Workshop Proceedings of the 18th International Conference on Computers in Education (ICCE 2010), pp. 361-365. Universiti Putra Malaysia, Malaysia (2010)

15. Alers, S., Barakova, E.I.: Multi-agent platform for development of educational games for children with autism. In: Games Innovations Conference, ICE-GIC 2009, pp. 47-53. International IEEE Consumer Electronics Society's (2009)

16. Bartneck, C., Hu, J.: Rapid Prototyping for Interactive Robots. In: The 8th Conference on Intelligent Autonomous Systems (IAS-8), pp. 136-145. IOS press, Amsterdam (2004)

17. Alers, S., Hu, J.: AdMoVeo: A Robotic Platform for Teaching Creative Programming to Designers. In: Chang, M., Kuo, R., Kinshuk, Chen, G.-D., Hirose, M. (eds.) Learning by Playing. LNCS, vol. 5670, pp. 410-421. Springer, Heidelberg (2009)

18. Sevarac, Z.: Neuro fuzzy reasoner for student modeling. In: Sixth International Conference on Advanced Learning Technologies, pp. 740-744. IEEE, Los Alamitos (2006) 